\title{
La Torre di San Francesco a Palmi nelle vedute di Edward Cheney del 1823: immagini di un baluardo scomparso del sistema difensivo vicereale della Calabria Ultra
}

The Tower of San Francesco in Palmi in the views of Edward Cheney of 1823: images of a disappeared bulwark of the viceregal defensive system of Calabria Ultra

\section{Maria Rossana Caniglia}

Università degli Studi Mediterranea di Reggio Calabria, Reggio Calabria, Italy, m.rossana_caniglia@libero.it

\begin{abstract}
To oppose the phenomenon of waves of Turks threatening the most exposed areas of the Kingdom of Naples, the viceregal government ordered from 1535 the construction of a continuous and articulated chain of defensive coastal towers.

In Calabria, on behalf of the Viceroy Pedro di Toledo, the Marquis Francesco Pignatelli developed a project to identify the most suitable and strategic sites where to build the towers along the Tyrrhenian and Ionian coasts. This network included 69 towers in Calabria Ultra and 33 in Calabria Citra, clearly visible from each other at a maximum distance of six thousand steps. Most of these towers have lost their original function over time, and after the taking of Algiers in 1830, some were used as customs posts or torri semaforiche, and then be permanently abandoned. Today almost all of them are ruins. The cartographic sources and above all the iconographic ones, testify the importance of this defensive system of towers suspended between the land and the sea and arranged one after the other, real sentinels of the Mediterranean.

On this occasion, the focus is on the Tower of San Francesco, was probably built in 1565, in Capo Barbi in Palmi, along the Tyrrhenian side between Reggio Calabria and Capo Vaticano. The bulwark was destroyed in 1956. The Tower of San Francesco, as evidenced by historical cartography and the views of Antonio Minasi in 1779 and Richard Keppel Craven in 1821, was portrayed in three drawings made by Edward Cheney during his travel to Calabria in May 1823. These views identify the characteristics of the architectural typology of the tower and the relationships with the town of Palmi; to relate it to the coastal towers of Pietre Nere (Taureana) and Capo Rocchi (Bagnara); and finally to the landscape of the Costa Viola up to the Strait of Messina.
\end{abstract}

Keywords: Calabria, Cheney, iconographic, tower.

\section{Introduzione}

"Per la secura custodia et defensione de le cita et terre de Marine del Regno da la invasione de corsari infideli" (Mafrici, 1980, p. 275) che il vicerè don Petro de Toledo (1484-1553) elaborò, dal 1535, un ambizioso progetto militare: una catena difensiva continua e articolata di torri lungo i litorali, e non solo, del Principato Citra, delle Calabrie e delle terre d'Otranto. Il Mediterraneo, infatti, già agli inizi del Cinquecento era teatro di numerose e frequenti incursioni da par- 
te dei turchi e dei corsari barbareschi che attaccavano, con effetto sorpresa, le coste più esposte del Mezzogiorno.

Durante il suo viceregno, dal 1532 al 1553, il Toledo emanò diversi ordinamenti sia sulle fortificazioni delle città e di interi territori (organizzazione dei cittadini e il loro armamento nella difesa dei litorali) sia sugli eventuali trasferimenti degli abitanti in luoghi più sicuri: "li casali dele tu cita et terre de marina per esserno aperti et non possernosi cossi de facile chiudere et fonicarnose de muraglia ad ciò in omne tempo se possano salvare et redure in securo se porra hordinare che tucti li habitanti de dicti casali [...] debiano intrare et habitare dentro le cita et terre murate" (Mafrici, 1980, p. 275).

Per verificare l'effettiva esecuzione di tali disposizioni e lo stato del sistema difensivo del Regno, il vicerè, incaricò delle ispezioni. La prima fu affidata l'11 aprile 1538 a Giovanni Maria Buzzaccarino, architetto militare padovano, con il preciso compito di "veda et proveda le fabriche, fortificacione et reparacione dele città et castelle demaniale $[\ldots]$, acciò se habia da exequire il lavoro, et bisognando fare altri designi li faccia et proveda in altre cose necessarie" (Mafrici, 1980, p. 277). E nel 1541 lo stesso Toledo intraprese un viaggio con l'intento di accertare le difficoltà e la lentezza con cui proseguiva il progetto. Al capitano Giovanni de Vergara gli fu commissionata, nel 1550 e nel 1551, la revisione di "tutte le Castelle et fortezze del presente regno tanto quelli che stanno in terre de marina como ancho dentro de terra" (Mafrici, 1980, p. 289).

Nello stesso anno, invece, Fabrizio Pignatelli, marchese di Cerchiara, fu inviato in Calabria con l'incarico di scegliere i luoghi più consoni alla costruzione delle strutture fortificate. Egli, infatti, "consigliò, e poi con l'approvazione dé ministri regij ordinò la fabrica delle sudette Torri; non tanto per una momentanea difesa, quanto perché l'una, qual prima scorgesse il pericolo, col fuoco, dimostrandolo all'altre, in meno di poche ore ne venisse avvisato tutto il Regno" (Fiore, 1691, p. 27).

La realizzazione dell'ambizioso progetto difensivo di don Petro de Toledo, nonostante i numerosi sforzi e l'ingente finanziamento, fu destinato a fallire. Bisognò aspettare il 1563 quando il vicerè don Parafan de Rivera (1509-1571) attuò un nuovo piano, organico e razionale, che potenziava e sviluppava sistematicamente quello del 1537: la dislocazione di torri in esatti punti, lungo $2200 \mathrm{~km}$ di costa, a vista l'una dall'altra, così da costituire una catena ininterrotta di fortificazioni. Le direttive emanate, inoltre, vietavano a privati la costruzione di postazioni difensive e ordinavano l'esproprio di quelle esistenti (Mafrici, 1980, 1995; Cataldo, 2014, p. 126).

La costruzione delle torri iniziò, quasi contemporaneamente, in tutto il regno, e si concluse nel 1570.

\section{Il sistema difensivo lungo il litorale della Costa Viola}

Nel 1564 in Calabria arrivò l'ordine per la costruzione delle torri marittime e l'11 marzo 1565 il Pignatelli comunicava al vicerè che la maggior parte delle postazioni erano già state realizzate. Affermazione che, secondo diversi studiosi, può essere considerata inattendibile ma Vittorio Faglia, in particolar modo, evidenzia che questa potrebbe risultare veritiera solo perché nel caso specifico della Calabria erano state utilizzate e rimesse in funzione molte delle torri preesistenti (angioine e aragonesi) riducendo le nuove a un numero limitato (Valente, 1960, p. 18; Mafrici, 1980, p. 45; Faglia, 1984, p. 61).

Prima degli interventi coordinati e unificati da parte del viceregno spagnolo, le due provincie Ultra e Citra e in alcuni casi anche le Università (comuni) e i feudatari, avevano avviato, nonostante i divieti, la costruzione di torri e di altri sistemi difensivi contro il minaccioso fenomeno piratesco.

"In mezzo a tante calamità, quelle che più facevano vivere in continuo timore $[\ldots]$, la gente della regione del versante occidentale dell'estrema Calabria, erano le piraterie dei Turchi e degli Algerini, [...]. La audacia dei corsari era tale, che di tratto in tratto venivano con le loro galee, di nottetempo, prendendo a scorta il promontorio del monte S. Elia; e si nascondevano in alcune insenature e grotte, fra le rocce del lido dell'altissima costiera a picco, interposta tra Gioia e Bagnara" (De Salvo, 1899, p. 144). 
Carlo Spinelli, duca e signore di Seminara, non solo ricostruì la città di Carlopoli, l'attuale Pal$\mathrm{mi}$, tra il 1559 e il 1566 , dopo che era stata forse in parte distrutta dalla devastazione di Dragut nel 1549, ma predispose anche una cinta muraria e, inoltre probabilmente coeve agli stessi anni furono innalzate le due torri di guardia lungo la Costa Viola: "di esse, quella più vicina alla città, ebbe il nome di San Francesco; l'altra su un ciglione, presso la chiesa di S. Fantino, fu detta di Pietre Nere, nome che ha pure la marina sottostante. Questa torre, sull'intonaco, che dal lato di ponente, le riveste la base, porta, segnata a graffio, la data 1565" (De Salvo, 1899, pp. 159-160).

La torre di San Francesco, ubicata a Capo Barbi, nella zona della Marinella, tra il monte Sant'Elia e lo scoglio Galera, lungo il versante tirrenico tra Capo Vaticano e Reggio Calabria (Fig. 1), era in collegamento a vista verso nord con la torre di Pietre Nere (a $5 \mathrm{~km}$ ) e verso sud con quella di Capo Rocchi a Bagnara (a $7 \mathrm{~km}$ ). Queste tre torri rientravano nella tipologia di quelle a pianta circolare piccola con base troncoconica e corpo slanciato, che in Calabria hanno avuto una maggiore diffusione soprattutto nel periodo angioino, rappresentando il nucleo più importante di tutta la difesa costiera (Faglia, 1984b, pp. 310-311, 335-338, 357, 375).

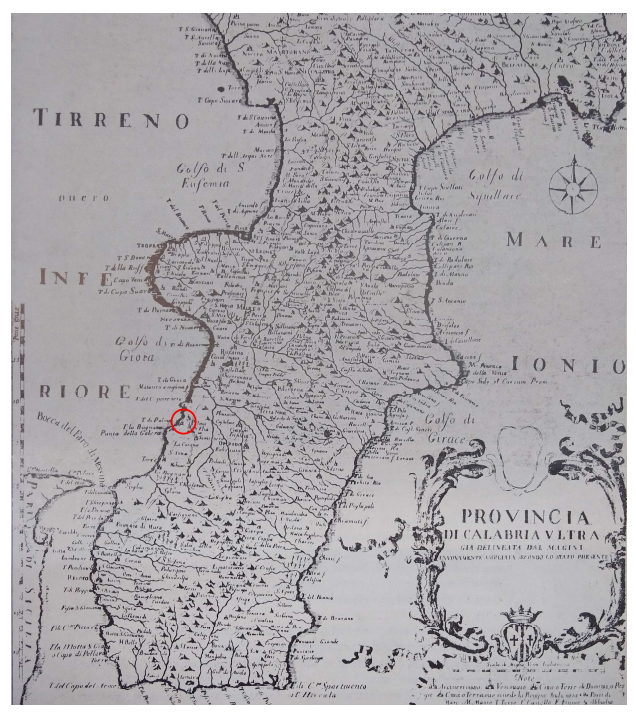

Fig. 1. Domenico De Rossi, Provincia di Calabria Citra già delineata dal Magini e nuovamente ampliata secondo lo stato presente, 1714. (Faglia, 1984, 35).
La torre di San Francesco nella toponomastica e nei vari documenti storici è identificata anche con le denominazioni di Palma, de Palma, Balbi, Capo Barbi, Belve; lo storpiamento e soprattutto l'alterazione radicale del nome hanno portato in alcuni casi a confondere la torre con altre strutture o a collocarla in un luogo diverso (Faglia, 1984a, pp. 75-76, 1984b, p. 375).

Le fonti bibliografiche fino a ora note sono esigue, discordanti e frammentarie, il tema in esame, di conseguenza, necessita ancora di ulteriori indagini e accurati approfondimenti critici.

\section{3. "Posta nel Capo de baluj nel territorio de Palmi": la torre di San Francesco}

Le vedute realizzate dai numerosi touristes, che dal Settecento in poi, intrapresero un viaggio in Calabria, rappresentano e raccontano città e territori prima delle progressive trasformazioni subite, dove in alcuni casi è mutata anche la caratterizzazione paesaggistica. In questa occasione i disegni di Edward Cheney diventano un supporto fondamentale: una fonte iconografica che testimonia la presenza della torre di San Francesco, oggi un baluardo scomparso (Fig. 2).

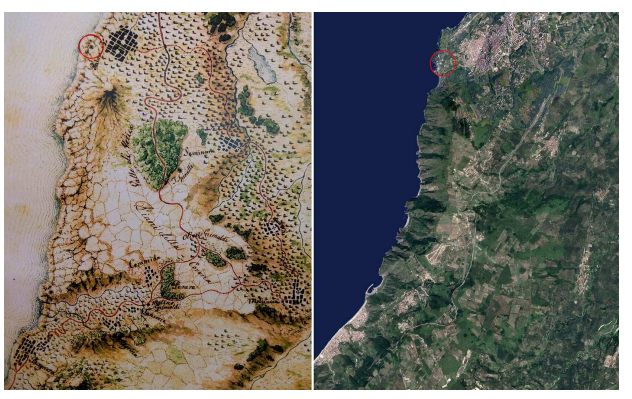

Fig. 2. Individuazione della torre di San Francesco. Confronto tra la Pianta dinotante la posizione di Melicuccà Palmi e Bagnara col paese corrispondente, 1835 (Cataldo, 2014) e lo stato attuale.

Cheney (1803-1884) colto letterario, mecenate e collezionista d'arte appartenente a una nobile famiglia inglese, decise di intraprendere, tra il 1823 e il 1825, insieme al fratello Robert-Henry (1801-1886), un viaggio nell'Italia meridionale: dalla Campania alla Sicilia, attraversando la Calabria, alla ricerca del tanto celebrato "paesaggio classico" (Caniglia, 2017). 


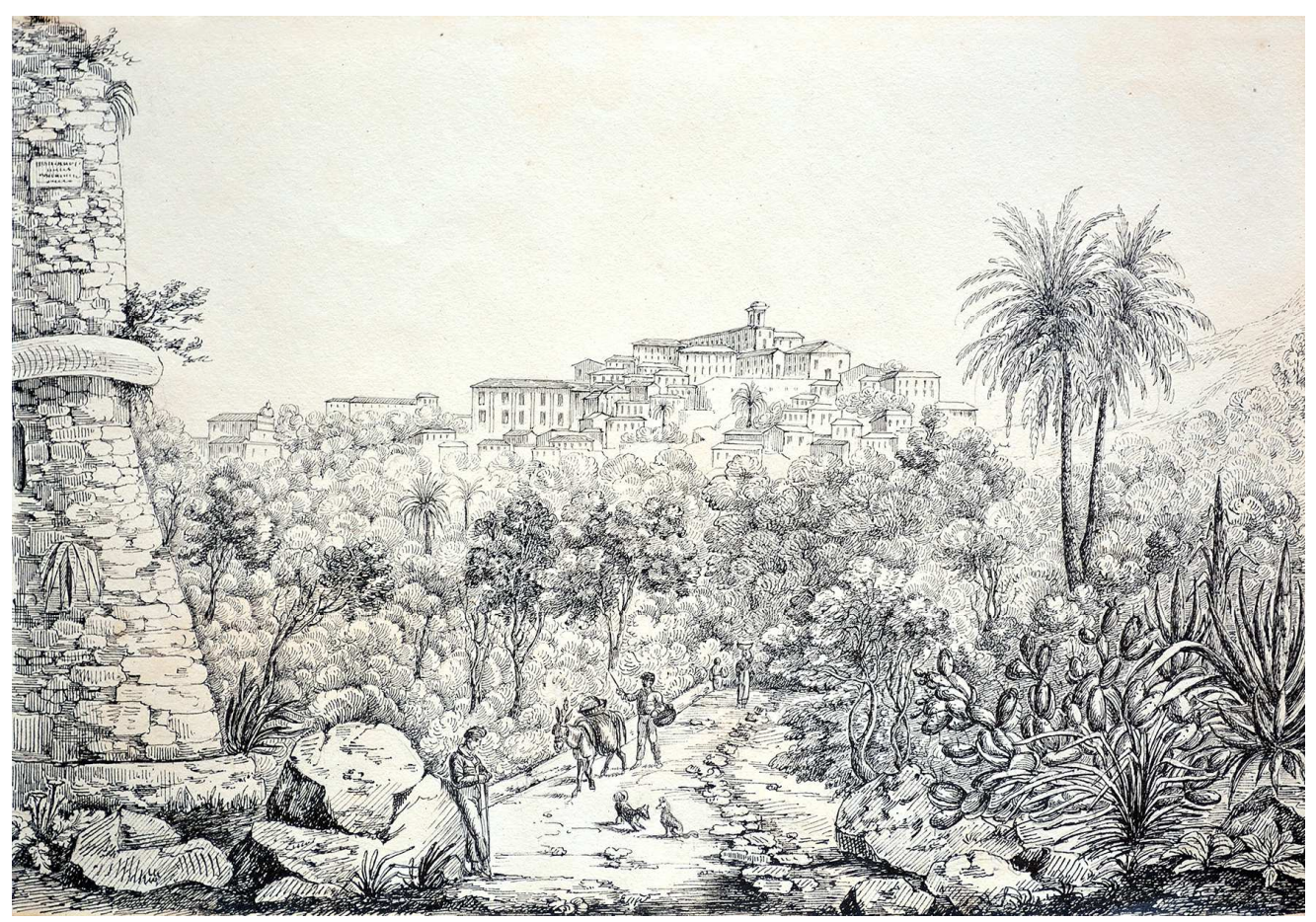

Fig. 3. Edward Cheney, Palmi Calabria Ultra, 15 maggio 1823, disegno a matita e china (riproduzione del disegno per gentile concessione dell'Istituto della Biblioteca Calabrese di Soriano Calabro).

In particolar modo l'itinerario percorso in Calabria è narrato dalle dodici vedute realizzate da Edward, che rappresentano i peculiari e suggestivi paesaggi di questa regione: dalle montagne del Pollino a quelle della Sila, dalla piana di Sibari alla costa tirrenica degli Dei fino ad arrivare alla Costa Viola. Paesaggi mutevoli dove la presenza dell'architettura, che in alcuni casi, forse quasi involontariamente, assume il ruolo di protagonista, s'intreccia con quella incontaminata e selvaggia della natura e viceversa. Il viaggio iniziò il 5 maggio 1823 con la tappa di Campotenese, nei pressi di Morano Calabro, in provincia di Cosenza e si concluse il 18 maggio con quella di Reggio Calabria, dopo aver visitato diversi luoghi e soggiornato in alcune città come Monteleone, l'attuale Vibo Valentia, e Palmi. Qui i fratelli Cheney arrivarono il 15 maggio.

Nella prima veduta Palmi Calabria Ultra l'attenzione di Edward è "catturata" da un antico percorso che collegava il centro urbano alla zona costiera della Marinella (Fig. 3).
Una porzione della torre in primo piano occupa il margine sinistro del foglio, mentre tutto attorno si sviluppa una rigogliosa vegetazione spontanea dalla quale emergono piante di vario genere come palme, agavi e fichi d'india, interrotta soltanto dalla strada animata dalla presenza di persone e alcuni animali. I piani prospettici sono in qualche modo connessi dal disegno della vegetazione che si espande fino al centro abitato, avvolgendolo e quasi nascondendo le sue architetture. Tra queste sono riconoscibili, a sinistra, la chiesa del Carmine, così come era prima di essere distrutta dal terremoto del 1894, accanto la chiesa dei Monaci o del Santissimo Crocifisso, mentre in alto spicca quello che potrebbe essere il vecchio campanile della chiesa del Soccorso. Inoltre, un altro elemento ben identificabile è il terrapieno, creato con l'accumulo delle macerie degli edifici demoliti dopo il disastroso terremoto del 1783, oggi la villa comunale Giuseppe Mazzini.

Questo disegno, rappresenta un significativo documento iconografico, non solo perché riproduce 
la struttura della torre, con la muratura in pietrame e gli elementi architettonici che ne caratterizzavano la tipologia; ma narra il rapporto urbano tra il baluardo difensivo e la città di Palmi, una visione prospettica così come doveva apparire in quegli anni prima delle diverse trasformazioni e della definitiva distruzione della torre nel 1956. In questa veduta, come in altre realizzate in Calabria, emerge la ferma volontà di Edward che fosse la realtà stessa, rappresentata dalle architetture e dai luoghi visitati, a diventare la chiave di lettura per far riscoprire la naturale ed equilibrata armonia del paesaggio.

Per la seconda veduta In the Golfo di Goija (Fig. 4) Edward si pose sempre vicino alla torre di San Francesco, ma cambiò punto di vista. Egli volse l'attenzione al tratto di costa calabrese che prolungandosi verso sud arrivava a scorgere Scilla, lasciando sullo sfondo la Sicilia oltre lo stretto di Messina con l'Etna fumante: probabilmente il panorama che stava ammirando il personaggio rappresentato seduto ai piedi della torre. In mare, anche se con un tratto appena accennato, s'intravedono i luntri, le tipiche barche usate per la pesca diurna del pesce spada.

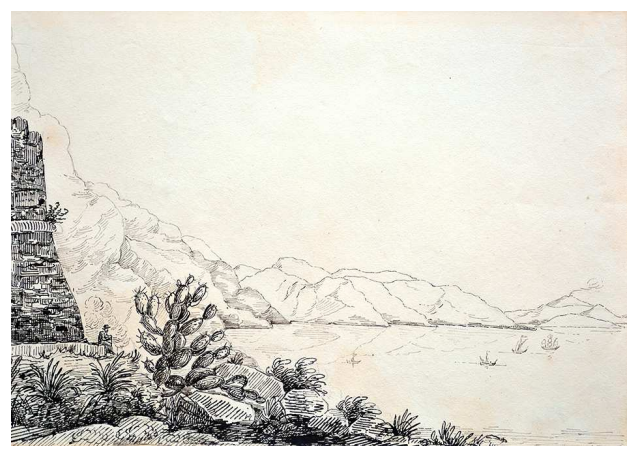

Fig. 4. Edward Cheney, In the Golfo di Goija, 15 maggio 1823 , disegno a matita e china (riproduzione del disegno per gentile concessione dell'Istituto della $\mathrm{Bi}$ blioteca Calabrese di Soriano Calabro).

Se in questa veduta, rispetto alla precedente, l'unica architettura presente è quella della torre, che con il ruolo da protagonista crea un legame diretto e armonioso con il peculiare paesaggio naturalistico circostante: quello calabrese della Costa Viola e lo skyline dello Stretto di Messina.
È necessario, invece, evidenziare che entrambi i disegni sono "animati" da personaggi che, oltre ad avere la funzione di illustrare scene di vita, dal punto di vista compositivo diventano il riferimento per ricondurre l'osservatore alla reale dimensione dell' architettura e della vegetazione rappresentate.

Confrontando la veduta ottocentesca con la situazione attuale, è stato possibile individuare l'originale punto di vista da cui il disegno fu tratto e costatare come siano riconoscibili, seppur filtrati dall'intermediazione dell'artista, i principali caratteri territoriali e paesaggistici rappresentati (Fig. 5).

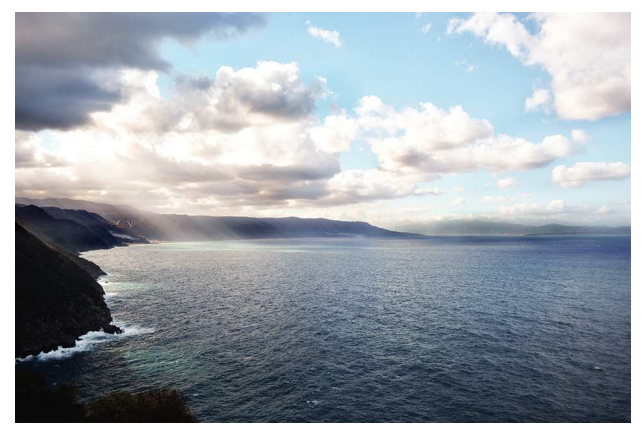

Fig. 5. La costa tirrenica vista da Palmi (Maria Rossana Caniglia, 2019).

Edward e Robert-Henry proseguirono il viaggio verso sud, percorrendo probabilmente la Strada Regia delle Calabrie, e il giorno successivo raggiunsero Bagnara. Qui Edward realizzò una veduta particolarmente suggestiva Bagnara in $\mathrm{Ca}$ labria Ultra. Il disegno illustra uno scorcio della Costa Viola inquadrata da sud verso nord, un punto di vista opposto rispetto al senso di percorrenza dell'itinerario. Nella composizione della veduta il Cheney pose in primo piano, evidenziandoli con tratto grafico fitto, preciso e chiaroscurato, i blocchi squadrati di antiche rovine e un tratto di muro in opus reticulatum, in parte ricoperti da una vegetazione in cui non si ritrova l'esuberanza registrata nelle altre vedute. Il segno diventa, invece, leggero ma incisivo nel raffigurare, in secondo piano, il luminoso tratto costiero a nord di Bagnara, identificato dal monte Sant'Elia, crinale costiero dell' Aspromonte, dalle due torri 


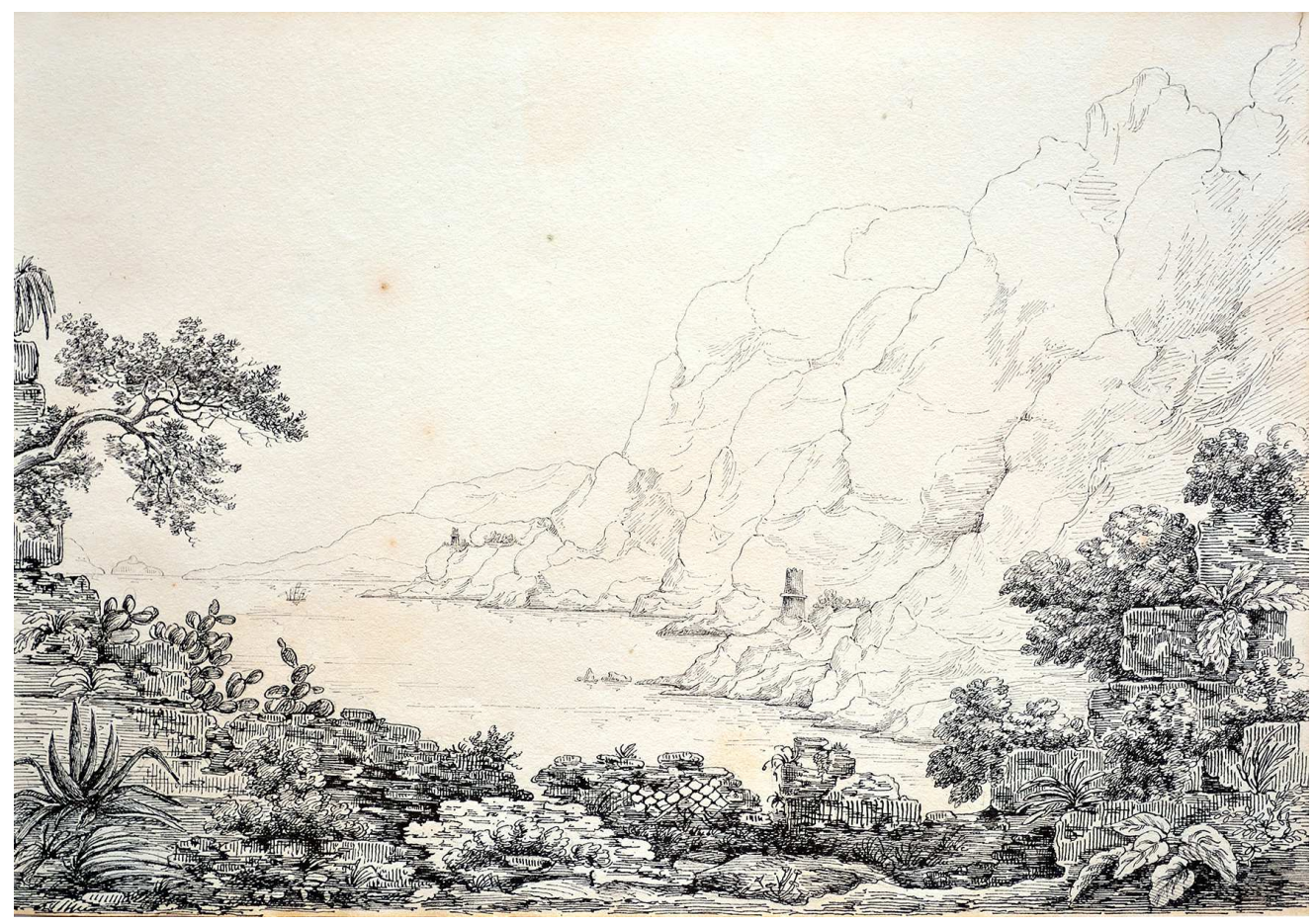

Fig. 6. Edward Cheney, Bagnara in Calabria Ultra, 16 maggio 1823, disegno a matita e china (riproduzione del disegno per gentile concessione dell' Istituto della Biblioteca Calabrese di Soriano Calabro).

di Capo Rocchi e di San Francesco e, in lontananza sullo sfondo il promontorio di Capo Vaticano (Fig. 6).

Questo disegno restituisce, in parte, quella catena ininterrotta di fortificazioni che il progetto difensivo dei vicerè aveva ideato, nel caso specifico il tratto costiero tra Palmi e Scilla, dove in pochi chilometri si trovavano quattro torri (e ne erano previste delle altre) sospese tra la terra e il mare e disposte una dietro l'altra, come delle vere e proprie sentinelle del Mediterraneo.

È interessante raffrontare questa veduta con quelle realizzate dall'illustratore Antonio Minasi (1736-1806), nel 1779, Prospetto del Faro di Messina, Riviera di Scilla, Costiera di Parma, e Spiaggia di Gioia (Fig. 7) e da Richard Keppel Craven (1779-1851), nel 1821, The entrance to the Faro of Messina (Fig. 8).

Il Minasi nella restituzione grafica della veduta fa emergere la forte appartenenza a quei luoghi, dove egli stesso viveva interpretando "quel senso di genius loci ancora intatto nella sua fresca e palpitante umanità naturalistica e di osservatore per nulla disincantato o neutro" (Principe, 1993, pp. 26, 79).

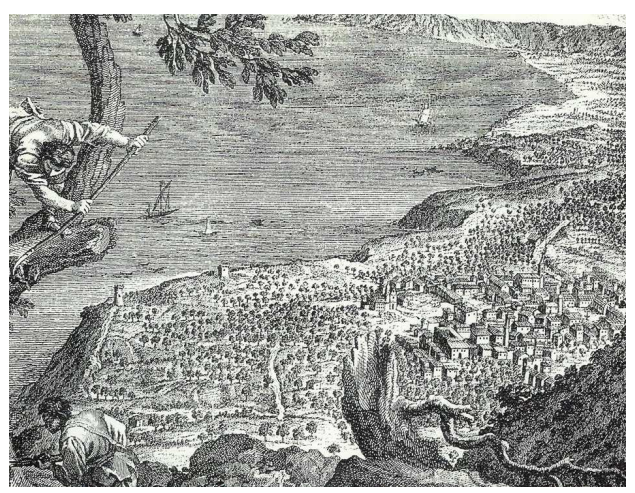

Fig. 7. Antonio Minasi, Prospetto del Faro di Messina, Riviera di Scilla, Costiera di Parma, e Spiaggia di Gioia, 1779, particolare (Principe, 1993, p. 79).

Keppel, invece, nel 1818 intraprese un viaggio nel sud dell'Italia e nel 1821 pubblicò il volume A Tour through the southern provinces of the 
kingdom of Naples. Arrivato a Palmi, descrisse quei luoghi in questo modo: "The space between the town and the edge of the rocks is prolonged in the shape of a promontory, and is the site of a watch-tower. The Lipari islands present them selves in front, and the view on each side is equally beautiful; that to the right commands the whole Gulf of Gioia as far as Cape Vaticano, while the other extends to the entrance of the Faro, and includes the Calabrian coast, with Scilla and Bagnara, as far as the Punta del Pezzo" (Keppel Craven, 1821, p. 291).

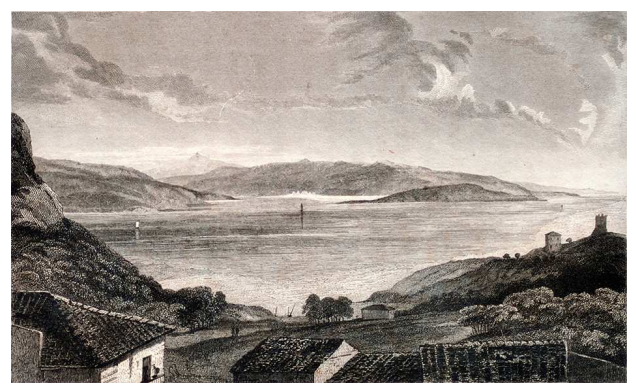

Fig. 8. Richard Keppel Craven, The entrance to the Faro of Messina, 1821 (Keppel Craven, 1821).

\section{Conclusioni}

La Calabria, tra tutto il regno vicereale, è il territorio con il maggior numero di torri: tra la metà e la fine del Seicento ne erano state censite 69 nella provincia Ultra e 33 in quella Citra (Algranati, 1957; Mafrici, 1978); il Quadro Generale delle torri marittime del litorale delle due Calabrie, edito nel 1820, elencava, invece, 150 torri; e infine dallo studio attento e scrupoloso condotto dal Faglia risultano in totale 144 strutture difensive (Faglia, 1984a, 1984b).

"Ora queste Torri, alcune già dirute, altre diroccate e distrutte, restano come una memoria storica ed un avanzo di antichità monumentale. I progressi moderni...le hanno fatte abbandonare per sempre, come avvisi e segnali dé nemici" (Valente, 1960, p. 113).

La maggior parte delle torri, già dal 1720, aveva perso la loro funzione originaria, e soprattutto dopo la battaglia di Algeri del 1830 con la quale si concluse il fenomeno della pirateria, alcune furono adibite a posti di dogana o a "torri semaforiche"; dal 1827 molte delle quali furono vendute ai privati e le altre definitivamente abbandonate (Mafrici, 1978; Maio, 1990, pp. 86-87). Oggi, tranne che per poche eccezioni, quasi tutte sono ridotte a poco più di un rudere.

\section{Bibliography}

Algranati, G. (1957). "Le torri marittime in Calabria nel periodo viceregnale", Calabria nobilissima, XI, 33, pp. 7377.

Bartone, F. (2013). Il Sublime e il Pittoresco. La Calabria nei disegni di Edward Cheney, Adhoc Edizioni, Vibo Valentia.

Caniglia, M.R. (2017). "L'Italia meridionale nei disegni di Edward e Robert-Henry Cheney (1823-1825)", in Belli, G.; Capano, F.; Pascariello, MI.I, eds., La città, il viaggio, il turismo. Percezione, produzione e trasformazione, CIRICE, Napoli, pp. 695-701.

Caniglia, M.R. (2019). "Il viaggio nell'Italia meridionale di Edward e Robert-Henry Cheney (1823-1825): la scoperta della Calabria, terra misteriosa e selvaggia”, in Mussari, B.; Scamardì, G., eds., Il Sud Italia: schizzi e appunti di viaggio. L'interpretazione dell'immagine, la ricerca di una identità, ArcHistoR Extra, 5, Supplemento di ArcHistoR, 11, pp. 348-395.

Carafa, R.; Calderazzi, A. (1999). La Calabria fortificata. Ricognizione e schedatura del territorio, Edizioni Mapograf, Vibo Valentia.

Cataldo, V. (2014). La frontiera di pietra. Torri, uomini e pirati nella Calabria moderna, Edizioni Scientifiche Italiane, Napoli

Cricrì, G. (2012). "La Torre ritrovata. Scoperti tre antichi disegni che ritraevano l'«osservatorio difensivo» cinquecentesco intitolato a San Francesco", Madreterra, 29, pp. 8-9. 
Faglia, V. (1984a). Tipologia delle torri costiere di avvistamento e segnalazione in Calabria Citra in Calabria Ultra dal XII secolo, Ricognizioni, Istituto Italiano dei Castelli, Roma, vol. 1.

Faglia, V. (1984b). Tipologia delle torri costiere di avvistamento e segnalazione in Calabria Citra in Calabria Ultra dal XII secolo, Ricognizioni, Istituto Italiano dei Castelli, Roma, vol. 2.

Fiore, P.G. (1691). Della Calabria illustrata, Napoli. Vol. I.

Giacomantonio R. (1988-1989). "Alcune osservazioni sulle torri costiere della fascia tirrenica della Calabria Citra e pastille sui rapport tra sistema difensivo costiero, le vie istmiche ed i centri urbani”, Calabria nobilissima, XLXLI, 88-89-90-91, pp. 73-92.

Keppel Craven, R. (1821). A Tour through the Southern Provinces of the Kingdom of Naples, Printed for Rodwell and Martin, London.

Liberti, R. (2000). "Pirateria e Guerra di Corsa”, Quaderni Mamertini, Litografia Diaco, Bovalino (Rc), 17.

Maio, D. De. (1990). Fanói. Calabria, Musulmani, Torri Costiere, Edizioni Bolis, Bergamo

Mafrici, M. (1978). "Il sistema difensivo costiero calabrese. Le torri”. Brutium, LVII, 4, pp. 12-16.

Mafrici, M. (1980). "Il sistema difensivo calabrese nell'età viceregnale”, Rivista storica calabrese, I, 1-2, pp. 29-52.

Mafrici, M. (1980). "Il sistema difensivo calabrese nell'età viceregnale", Rivista storica calabrese, I, 3-4, pp. 271302.

Mafrici, M. (1988-1989). "La difesa delle coste meridionali nei secoli XVI-XVII: tecnici e tecnologie", in Placanica, A., ed., Annali del Centro Studi «Antonio Genovesi» per la storia economica e sociale, Edizioni Scientifiche Italiane, Napoli, vol. 1, pp. 31-106.

Mafrici, M. (1995). Mezzogiorno e pirateria nell'età moderna (secoli XVI-XVIII), Edizioni Scientifiche Italiane, Napoli.

Martorano, F. (2009). "Territorio e città nella politica dei Carafa di Roccella e degli Spinelli di Seminara e Ricca tra Cinque e Seicento", in Anselmi, A., ed., La Calabria del viceregno spagnolo: storia arte architettura e urbanistica, Gangemi, Roma, pp. 227-247.

Martorano, F. (2015). Progettare la difesa, rappresentare il territorio. Il Codice Romano Carratelli e la Fortificazione nel Mediterraneo secoli XVI-XVII, Edizioni Centro Stampa d'Ateneo, Reggio Calabria.

Mazzella Napolitano, S. (1601). Descrittione del Regno di Napoli, Ad istanza di Gio, Battista Cappello, Napoli.

Principe, I. (1993). Paesaggi e vedute della Calabria nella raccolta Zerbi, Edizioni Mapograf, Vibo Valentia.

Romeo, V. (2016). Sentinelle dimenticate. Il sistema collaborante di avvistamento e difesa della Calabria nel XVI secolo. Torri costiere e fortezze lungo la costa tirrenica, Aracne, Canterano, vol. 2.

Salvo, A. De. (1899). Ricerche e studi storici intorno a Palmi, Seminara e Gioia Tauro, Tip. Giuseppe Lopresti, Palmi.

Valensise, F. (2007). "L'organizzazione territoriale dello Stretto nell'iconografia di Antonio Minasi”, in Paola, F. Di, ed., La città per parti. Progetti urbanistici per l'area metropolitana dello Stretto, Centro stampa d'ateneo, Reggio Calabria, pp. 225-245.

Valente, G. (1960). Le torri costiere della Calabria, Tipografia-Linotypia Eredi Serafino, Cosenza. 